\title{
Acute Myocardial Infarction Associated with Trousseau's Syndrome: Case Report and Review of the Literature
}

S Stojnev ${ }^{1}$, M Krstić $c^{1,2}$, I Ilić ${ }^{1,2}$, I Petković 3 , S Stojanović ${ }^{1}$, P Cvetković ${ }^{4}$, P Randjelović ${ }^{1}$, LJ Veličković $^{1,2}$

\begin{abstract}
Objective: Trousseau's syndrome, malignancy associated hypercoagulability, comprises polymorphic spectrum of arterial and venous thrombotic disorders in setting of neoplastic disease. We suspected that acute myocardial infarction in a patient with low cardiovascular risk and suspicious lung infiltration might be a presentation of Trousseau's syndrome.

Methods: While waiting for diagnostic bronchoscopy, 49-year-old man with radiologically detected abnormal shadow in right lung hilum, suddenly developed myocardial infarction. The patient was treated and stabilized, however he died four days later, and clinical autopsy was performed.

Results: Post mortem results revealed poorly differentiated lung adenocarcinoma in advanced stage. Blood clots were found in medium and small branches of coronary blood vessels not only in the field of myocardial infarction, but also in surrounding non-necrotic muscle. Ischemic infarcts were found in kidney and spleen. Occlusive thrombi and thromboemboli were found in veins and arteries of medium and small caliber in visceral organs, and in venous plexuses of the pelvis.

Conclusion: Cancer associated hypercoagulability presented as myocardial infarction is very rarely encountered in clinical practice. Trousseau's syndrome as underlying cause of arterial thrombosis should be considered and investigated, especially for patients without clinical evidence of atherosclerosis.
\end{abstract}

Keywords: Trousseau's syndrome, myocardial infarction, lung adenocarcinoma, hypercoagulability

From: ${ }^{1}$ Faculty of Medicine, University of Nis, Zorana Djindjica Blvd 81, 18000 Nis, Serbia. ${ }^{2}$ Center for Pathology, Clinical Center Nis, Zorana Djindjica Blvd 48, 18000 Nis, Serbia. ${ }^{3}$ Clinic for Oncology, Clinical Center Nis, Zorana Djindjica Blvd 48, 18000 Nis, Serbia. ${ }^{4}$ Clinic for Cardiology, Clinical Center Nis, Zorana Djindjica Blvd 48, 18000 Nis, Serbia

Correspondence: Dr S Stojnev, Faculty of Medicine, University of Nis, Zorana Djindjica Blvd 81, 18000 Nis, Serbia. Email: slavicastojnev@gmail.com; slavica.stojnev@medfak.ni.ac.rs 


\section{INTRODUCTION}

Trousseau's syndrome (TS) is most commonly defined as hypercoagulability and spontaneous thrombotic events associated with cancer. Pathogenesis of malignancy related coagulation disorder has not been completely elucidated, but is likely multifactorial and involves multiple mechanisms and interplaying pathways $(1,2)$. Trousseau's syndrome was initially described by French internist Armand Trousseau in 1860s (3), who, ominously coincidentally, recognized this foretelling sign of visceral malignancy in himself and soon after succumbed to a gastric cancer.

TS often precedes the diagnosis of occult or unrecognized visceral malignancy or it presents during the diagnosis of cancer. Underlying cancer was detected in almost $10 \%$ of patients with symptomatic idiopathic thromboembolism (4). Cancers associated with TS are usually diagnosed in advanced stage, have poor prognosis and decreased survival, which may be linked to their aggressive biology $(2,5)$.

TS comprises any kind of coagulopathy linked to malignant disease $(2,6,7)$. In clinical practice, TS is most frequently presented in a form of deep vein thromboses and subsequent thromboembolism, however arterial thrombotic events are not rare $(7,8)$. Concurrent venous and arterial thromboses may be triggered by stimuli that activate coagulation and inflammatory pathways in whole circulation system.

Thrombotic events have the highest incidence in patients with lung cancer, probably due to high prevalence of pulmonary malignancy and its biological properties. Patients with non small cell lung cancer (NSCLC), especially adenocarcinoma, are at higher risk than those with small cell cancer histology $(5,9)$.

The effect of thrombotic events can be devastating in cancer patients: it may delay, hinder or preclude chemotherapy, decrease quality of life, increase health care and social 
burden and, as in herein described example, impose as a life threatening complication leading to death of the patient $(1,9)$.

Here we describe a rare case of TS presenting as acute myocardial infarction (AMI), associated with lung adenocarcinoma, which was diagnosed postmortem.

\section{CASE REPORT}

A 49 -year-old man with abnormal $15 \mathrm{~mm}$ shadow in right lung hilum on a chest X-ray was admitted to our hospital for diagnostic bronchoscopy. The patient had unremarkable medical and family history and was a non-smoker. He had no history of hypertension, coronary artery disease, hyperlipidemia, and use of illicit drugs. He reported significant weight loss during the last two months and occasional cough.

The physical examination revealed emaciated male patient, with stable vital signs, afebrile, with no other significant abnormalities. His laboratory tests revealed anemia (erythrocytes $\left.=3.15 \times 10^{6} / \mu \mathrm{L}, \mathrm{Hb}=8 \mathrm{~g} / \mathrm{dl}, \mathrm{Hct}=0.256\right)$, leukocytosis $\left(22.6 \times 10^{3} / \mu \mathrm{L}\right)$, and normal platelet count $\left(174 \times 10^{3} / \mu \mathrm{L}\right)$. Serum enzymes were significantly elevated (LDH=3143U/L, $\mathrm{CRP}=250 \mathrm{nmol} / \mathrm{L}, \mathrm{GGT}=199 \mathrm{U} / \mathrm{L})$. Coagulation parameters demonstrated highly increased Ddimer $(5250 \mathrm{ng} / \mathrm{mL}$, normal value $<250 \mathrm{ng} / \mathrm{mL}$ ), fibrinogen $=5.49 \mathrm{~g} / \mathrm{L}$ (reference range 1.7 $4.0 \mathrm{~g} / \mathrm{L})$, consistent with coagulopathy. Prothrombin time was $5.2 \mathrm{~s}$, and activated partial thromboplastin time $26.5 \mathrm{~s}$, both within normal range.

Two days after admission, the patient developed a massive acute myocardial infarction. Urgent electrocardiogram indicated myocardial anterior wall ischemia with ST segment elevation in precordial leads. The patient was urgently referred to coronary unit, where he received immediate care and as treated with percutanous coronary angioplasty. He was hemodynamically stabilized and returned to the hospital. The patient received anticoagulation drug Warfarin and antiaggregants therapy, acetylsalicylic acid and 
clopidogrel. Four days after the AMI, before diagnostic bronchoscopy could be performed, the patient died during sleep.

The autopsy revealed poorly differentiated adenocarcinoma of the central perihilar region of the right lung with extensive infiltration and enlargement of pulmonal, hilar and ipsilateral mediastinal lymph nodes. Besides the mediastinal encroachment, a $20 \mathrm{~mm}$ large neoplastic lesion was present on the posterior lateral wall of the right hemithorax, besides the vertebral column. Metastatic deposits were found in left suprarenal gland and in the left kidney in a form of nodular, seemingly well circumscribed masses. No sign of neoplastic process was found in the liver and contralateral lung.

Occlusive thrombi and thromboemboli were found in veins and arteries of medium and small caliber in visceral organs, and in venous plexuses of the pelvis. In the heart, blood clots formed predominantly of fibrin and in lesser extent of entrapped blood elements were found in medium and small coronary artery branches. This finding was prominent in the field of infarction, where necrosis of cardiomyocites was histologically obvious, but also in blood vessels of surrounding non-necrotic muscle. Verrucous thrombotic masses were found adhered to valvular endocardium of aortic valve. Pathohistological exam revealed that wedge shaped white-grayish fields found in left kidney and spleen represented coagulative necrosis, inflammatory infiltrate and stasis, consistent with recent ischemic infarction of these organs.

\section{DISCUSSION}

Trousseau's syndrome implies thrombotic problems that transpire in the setting of an occult or a recently diagnosed cancer and cannot be explained by any other obvious factor $(1,2)$. In a prospective randomized study of patients with idiopathic venous thromboembolism, an extensive screening for occult malignancy resulted in significantly reduced time to diagnosis 
and identification of cancer in an earlier stage (4). However, the impact of early detection of cancer on prognosis and quality of life remains unclear.

The frequency of thrombotic events among the patients with lung cancer is variable and influenced by numerous factors, but is generally higher in patients with NSCLC compared to SCLC, especially in those with adenocarcinoma histology. The incidence of VTE varies in patients with NSCLC, from $0 \%$ in early stage NSCLC to $7.9 \%$ in those with advanced disease who were treated with chemotherapy, and is fourfold higher in metastatic disease $(5,9)$. In recent report of four TS cases associated with stage IV lung adenocarcinoma, clinical manifestations included brain infarction, deep vein thrombosis of popliteal veins and occlusion of pulmonary artery, while laboratory findings shown elevation of fibrinogen degradation products, D-dimer, tissue factor and sialylated carbohydrate antigen KL-6 (MUC1) (10).

Patient with advanced lung adenocarcinoma, who developed non-bacterial thrombotic endocarditis, pulmonary embolism and low-grade disseminated intravascular coagulation was successfully treated with heparin and recombinant human soluble thrombomodulin, which enabled the patient to endure the following combination chemotherapy (6). In present report, we found thrombotic formations on aortic valvular cusps which may be morphological presentation of marantic nonbacterial thrombotic endocarditis. Infarcts of the kidney and spleen probably developed as a consequence of tromboemboli that arose from these vegetations. Recently, spontaneous thrombosis of internal jugular vein has been described in patient with NSCLC (11). This supports the notion that TS can manifest as very diverse and polymorphic spectrum of thrombotic disorders associated with malignancy.

In a report describing AMI and ischemic stroke in patient with NSCLC, ischemic events were attributed to chemotherapeutic agent vinorelbine (12). However, AMI in our patients occurred in setting of low cardiovascular risk profile, without clinical signs of 
atherosclerosis, and was not related to therapy. Pathohistological exam revealed blood clots throughout the circulation and verified multiple infarcts in visceral organs. Finding of microthrombi in preserved myocardium implies that thrombosis of coronary vessels was not merely a consequence of infarction. Clustering of an AMI and deep vein thrombosis educed comprehensive investigation of occult malignancy in a female patient without clinical signs of atherosclerosis (13). Clinical search resulted in discovery of adnexal mass which was pathologically diagnosed as mucin-secreting ovarian adenocarcinoma.

Factors that contribute to and cause hypercoagulability may be clustered as patient-, cancer-, and treatment-related (9). Advanced age, prior thrombotic event, comorbidities, and poor performance status often seen in cancer patients (patient-related factors) may increase the risk for thrombotic event, as well as surgical procedures, either diagnostic or therapeutic, and chemotherapy $(5,9)$. In a prospective study that included advanced stage NSCLC patients treated with cisplatin and gemcitabine, adverse vascular event were recorded in $17.6 \%$ of patients: 12 venous and 10 arterial, including two myocardial infarctions (14). New antiangiogenic drugs have also been associated with increased risk of vascular thrombosis $(15)$.

Related factors including tumor histology and stage significantly predispose vascular events. The risk of thrombosis is increased in adenocarcinomas of various locations, emphasizing the glandular tumor histology as important factor. Mucin-producing adenocarcinomas of the lung, pancreas, stomach are considered the most important triggers of hypercoagulability in malignant disease $(5,16)$. In patients with adenocarcinoma, particularly mucin producing adenocarcinoma, there is a constant tumor shedding resulting in presence of altered, abnormal mucins in the circulation $(1,16)$. Small amounts of mucins that escape liver clearance can interact with platelet P-selectin and leukocyte L-selectins, triggering the 
platelet aggregation without necessary activation of thrombin, which leads to rapid formation of platelet-rich microthrombi (16).

Emerging evidence suggests that cancer associated thrombosis is caused by overexpression of tissue factor $(\mathrm{TF})(1,9,17)$. Thrombin generated in response to $\mathrm{TF}$ activates platelets and mediates further events in coagulation cascade, finally leading to fibrin deposition and clot formation. Moreover, $\mathrm{TF}$ and thrombin mediate various signaling pathways with a role in angiogenesis, tumor growth, cell migration and metastasis development. In cancer patients, TF is overexpressed by the tumor cells, and as a response of host tissues to inflammation and damage caused by tumor growth. TF elevation has been linked to reduced survival in many malignancies, including lung carcinoma $(9,17)$. Recent study found that high levels of alternatively spliced TF, which lacks transmembrane domain and is thus solubile and present in circulation, have been associated with poor prognosis in NSCLC (18).

Complex mechanisms of cancer coagulopathy may be genetically modulated by driver mutations that alter the expression of TF and other proteins involved in hemostasis (19). Activation of MET oncogene upregulates transcription of plasminogen activator inhibitor type1 (PAI-1), and cyclooxigenase-2, and both support thrombohaemorrhagic phenotype in vivo (20). The EPICOR study identified PAI-1 as independent and common risk factor for cancer and ischemic cardiovascular disease, approximating the possible molecular link between mechanisms of thrombosis and tumorigenesis (21). In addition, recent study identified K-RAS mutation as a driver of thromboembolic risk in NSCLC patients (22).

Thrombotic events have negative impact on morbidity and mortality in cancer patients $(5,14)$. Although there is no established medical protocol for managing Trousseau's syndrome in cancer patients, it has been suggested that intensive anticoagulant treatment may provide significant benefits. After AMI our patient was treated with warfarin ant 
antiaggregants, however intravascular thrombosis was not prevented. Treatment of thrombotic events in lung cancer patients is preferably conducted by low molecular weight heparin, but therapy decision should be individualized $(9,23)$. Timely and adequate therapy of coagulation disorders improves patients' condition, prevents possibly deadly thromboembolic complications and enables the patient to endure chemotherapy. Exclusively the reduction of tumor, usually achieved by chemotherapy, leads to resolution of Trousseau's syndrome.

In conclusion, although the relationship between malignancy and thrombotic events is well known, even suspected, undiagnosed cancer should urge the clinician to closely monitor the coagulation status of the patient and consider antithrombotic prophylaxis. Trousseau's syndrome is not rare, however the presentation with AMI is very seldom encountered in clinical practice. For patients without clinical evidence of atherosclerosis, hypercoagulability associated with cancer as underlying cause of AMI should be considered and investigated.

\section{ACKNOWLEDGEMENTS}

This study was supported in part by a Grant No 175092 from the Ministry of Education Science and Technological Development of Serbia. 


\section{REFERENCES}

1. Varki A. Trousseau's syndrome: multiple definitions and multiple mechanisms. Blood 2007; 110: 1723-9.

2. Dammacco F, Vacca A, Procaccio P, Ria R, Marech I, Racanelli V. Cancer-related coagulopathy (Trousseau's syndrome): review of the literature and experience of a single center of internal medicine. Clin Exp Med 2013; 13: 85-97.

3. Trousseau A. Phlegmasia alba dolens. Clinique Médicale de l'Hotel-Dieu de Paris $1868 ; 3: 43$.

4. Piccioli A, Lensing AW, Prins MH, Falanga A, Scannapieco GL, Ieran M, et al. Extensive screening for occult malignant disease in idiopathic venous thromboembolism: a prospective randomized clinical trial. J Thromb Haemost 2004; 2: 884-9.

5. Chew HK, Davies AM, Wun T, Harvey D, Zhou H, White RH. The incidence of venous thromboembolism among patients with primary lung cancer. J Thromb Haemost 2008; 6: 601-8.

6. Yoshii Y, Numata T, Ishitobi W, Takahashi N, Wakui H, Kojima J, et al. Lung adenocarcinoma complicated by Trousseau's syndrome successfully treated by a combination of anticoagulant therapy and chemotherapy. Intern Med 2014; 53: 1835 9.

7. Prandoni P, Piovella C, Pesavento R. Venous thromboembolism and arterial complications. Semin Respir Crit Care Med 2012; 33: 205-10.

8. Blann AD, Dunmore S. Arterial and venous thrombosis in cancer patients. Cardiol Res Pract 2011; 2011: 394740.

9. Corrales-Rodriguez L, Blais N. Lung cancer associated venous thromboembolic disease: a comprehensive review. Lung Cancer 2012; 75: 1-8. 
10. Tachihara M, Nikaido T, Wang X, Sato Y, Ishii T, Saito K, et al. Four cases of Trousseau's syndrome associated with lung adenocarcinoma. Intern Med 2012; 51: 1099-102.

11. Kunimasa K, Korogi Y, Okamoto Y, Ishida T. Spontaneous internal jugular vein thrombosis associated with lung cancer. Intern Med 2013; 52: 1849.

12. Zerna C, Guenther M, Folprecht G, Puetz V. Acute ischaemic stroke and myocardial infarction after chemotherapy with vinorelbine for non-small cell lung cancer: a case report. J Chemother 2015; 11: 1973947814 Y0000000232.

13. CM Yang, MY Hsieh, L Lin, CC Wu. Acute myocardial infarction in association with occult ovarian cancer. Acta Cardiol Sin 2013; 29: 102-6.

14. Numico G, Garrone O, Dongiovanni V, Silvestris N, Colantonio I, Di Costanzo G, et al. Prospective evaluation of major vascular events in patients with nonsmall cell lung carcinoma treated with cisplatin and gemcitabine. Cancer 2005; 103: 994-9.

15. Choueiri TK, Schutz FA, Je Y, Rosenberg JE, Bellmunt J. Risk of arterial thromboembolic events with sunitinib and sorafenib: a systematic review and metaanalysis of clinical trials. J Clin Oncol 2010; 28: 2280-5.

16. Wahrenbrock M, Borsig L, Le D, Varki N, Varki A. Selectin-mucin interactions as a probable molecular explanation for the association of Trousseau syndrome with mucinous adenocarcinomas. J Clin Invest 2003; 112: 853-62.

17. Rickles FR, Patierno S, Fernandez PM. Tissue factor, thrombin, and cancer. Chest 2003; 124 (3 Suppl): 58S-68S.

18. Rollin J, Regina S, Gruel Y. Tumor expression of alternatively spliced tissue factor is a prognostic marker in non-small cell lung cancer. J Thromb Haemost 2010; 8: 60710. 
19. D'Asti E, Magnus N, Meehan B, Garnier D, Rak J. Genetic basis of thrombosis in cancer. Semin Thromb Hemost 2014; 40: 284-95.

20. Boccaccio C, Sabatino G, Medico E, Girolami F, Follenzi A, Reato G, et al. The MET oncogene drives a genetic programme linking cancer to haemostasis. Nature 2005; 434: 396-400.

21. Iacoviello L, Agnoli C, De Curtis A, di Castelnuovo A, Giurdanella MC, Krogh V, et al. Type 1 plasminogen activator inhibitor as a common risk factor for cancer and ischaemic vascular disease: the EPICOR study. BMJ Open 2013; 3: e003725.

22. Corrales-Rodriguez L, Soulières D, Weng X, Tehfe M, Florescu M, Blais N. Mutations in NSCLC and their link with lung cancer-associated thrombosis: a casecontrol study. Thromb Res 2014; 133: 48-51.

23. Ben-Aharon I, Stemmer SM, Leibovici L, Shpilberg O, Sulkes A, Gafter-Gvili A. Low molecular weight heparin (LMWH) for primary thrombo-prophylaxis in patients with solid malignancies - systematic review and meta-analysis. Acta Oncol 2014; 53: $1230-7$. 

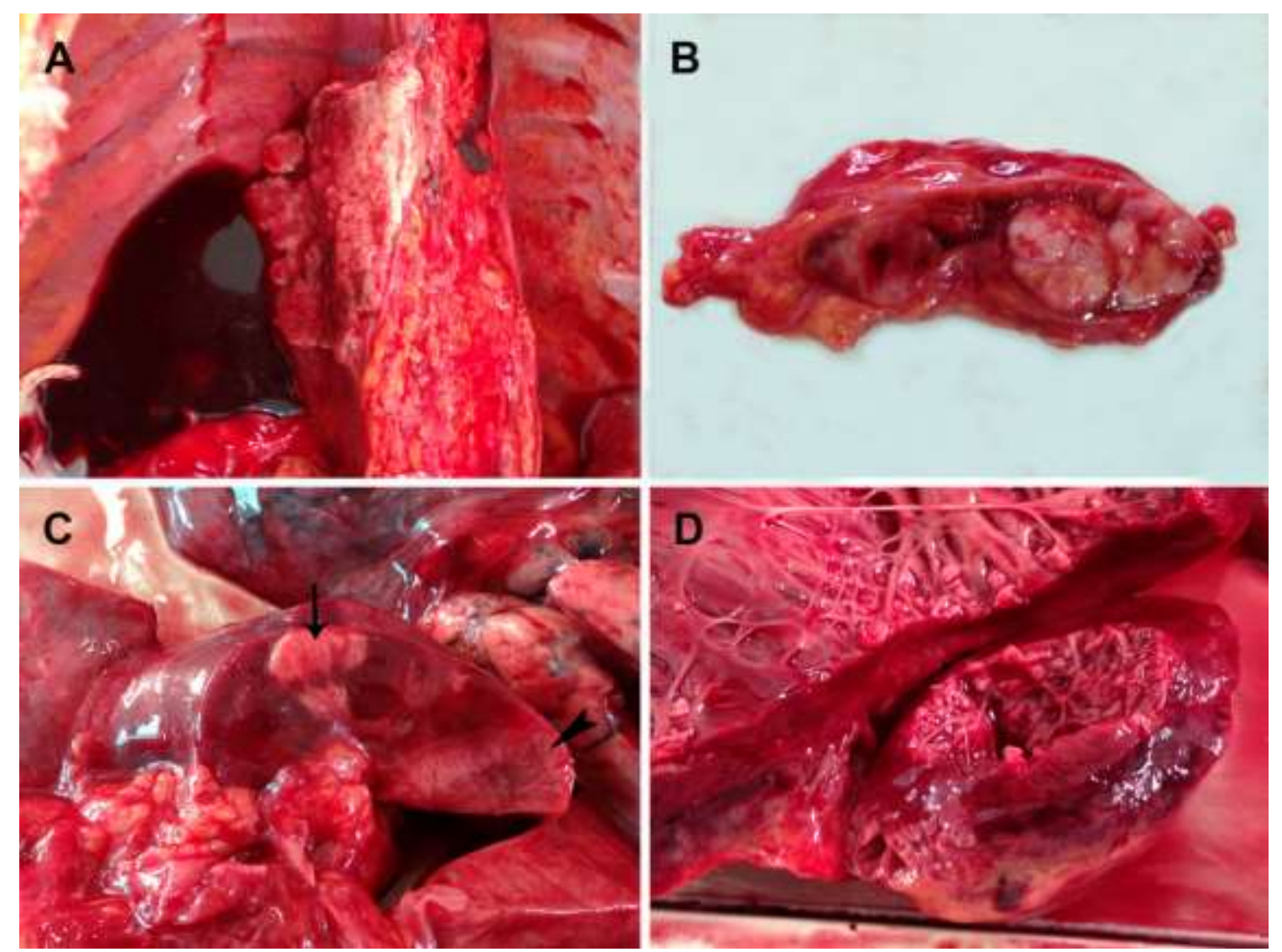

Fig 1: Gross appearance of lung adenocarcinoma spreading paravertebrally to thoracic wall (A); metastasis in left adrenal gland (B); metastasis (arrowhead) and infarct (arrow) in the left kidney (C); massive acute myocardial infarction (D). 


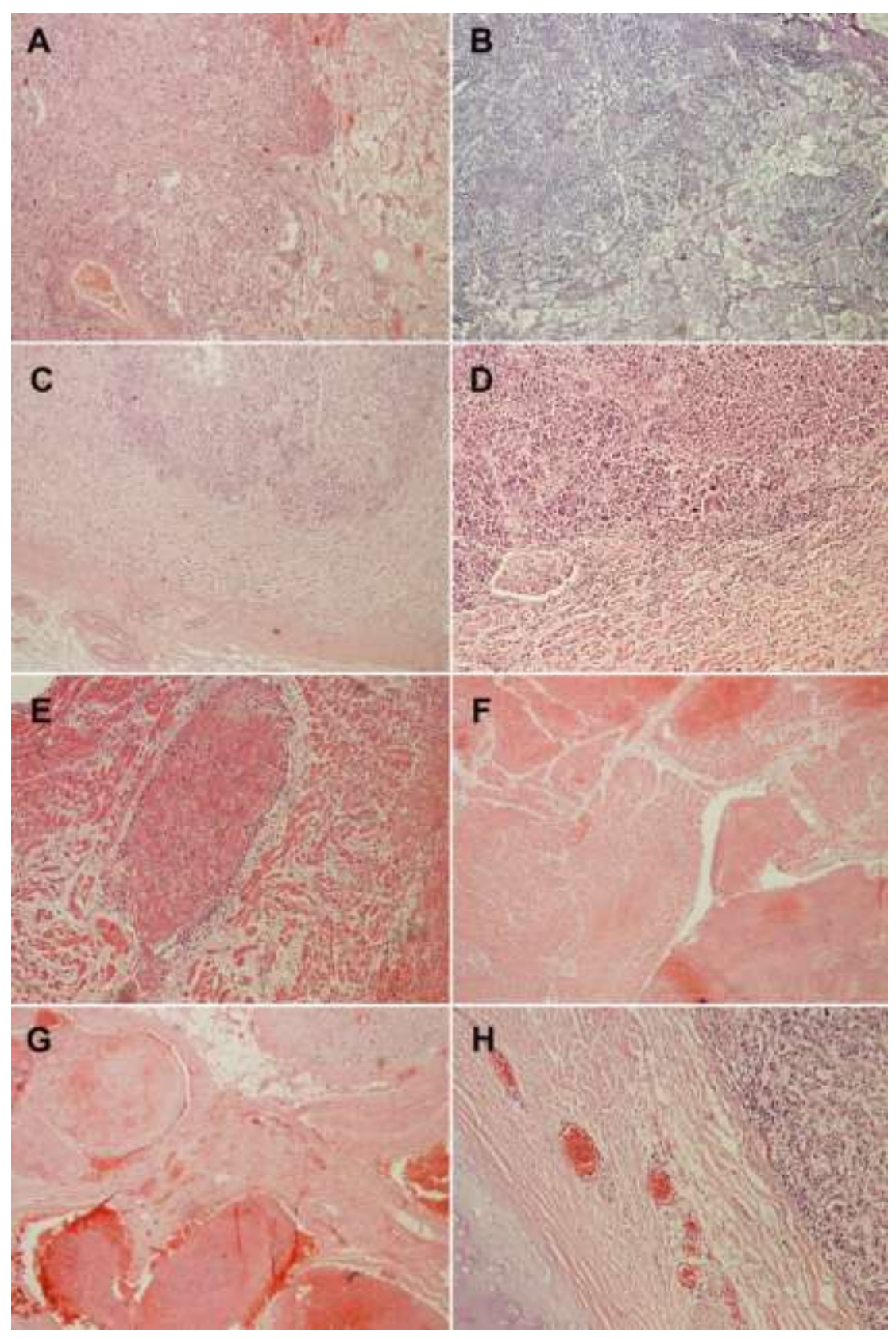

Fig 2: Representative photomicrographs of postmortem tissue showing poorly differentiated lung adenocarcinoma (A) HE stain, and (B) AB-PAS histochemical stain; metastasis to adrenal gland (C) and kidney (D); thrombotic mass in coronary vessel (E) and mural thrombosis of endocardium $(\mathrm{F})$; extensive thrombosis of periprostatic venous plexus $(\mathrm{G})$; adenocarcinoma spreading adjacent to right primary bronchus $(\mathrm{H})$. 